\title{
Precise IR-Based Temperature Measuring - a Case Study for the Automatic Health Monitoring of Dairy Cows
}

\author{
Wirthgen, Tom¹; Zipser, Stephan ${ }^{1}$; Geidel, Steffi'; Franze, Ulrike ${ }^{2}$ \\ ${ }^{1}$ Fraunhofer Institute for Transportation and Infrastructure Systems, Zeunerstr. 38, 01069 Dresden \\ ${ }^{2}$ University of Applied Sciences Dresden, Faculty Agriculture/ Landscape management, Pillnitzer Platz 2, 01326 Dresden
}

\section{Motivation and Introduction}

In modern livestock farming more and more processes are automated, because stock size is increased while at the same time the number of employees stagnates. These circumstances raise the requirements for stock management, especially health management. Therefore automatic health monitoring systems are needed to fulfil the rising needs for animal welfare and food safety.

Absolute and relative body temperature changes can be symptoms of diseases like certain inflammations. The temperature is often measured with conventional infrared thermography (IRT). However, the precision and the reproducibility are limited by the IRT device, physiological reactions and individual cycles like the circadian rhythm. To minimize these known disturbing effects standardized IRT diagnostic procedures were developed for the human medicine (constant environmental conditions, defined patient instruction, measuring system, image analysis, etc.). Due to practical restrictions these cannot be transferred to veterinary applications.

Veterinary IRT is applied in the field of academic investigations as well as medical praxis (horse riding, zoological garden) [11]. In recent studies usually calibrated hand held multi purpose IR cameras are used to take few single images from different perspectives. The correction of background radiation is only done in few studies. The measurement uncertainty is not quantified systematically in most studies $[1,4,8-10]$. The common measuring configuration allows intra-image differential measurements with an uncertainty near the thermal resolution of the camera. For time series or inter-image comparisons an in-depth analysis of the uncertainty in relation to the diagnostic statistics is necessary. Under good conditions the expected uncertainty is in range of the camera uncertainty and in many cases greater than the diagnostic temperature difference. This makes it difficult to evaluate the potentials and limits of veterinary IRT. The interdisciplinary project VIONA (www.viona-system.net) was established to investigate IRT for dairy cows under real farming conditions. The project focuses on the automatic detection of frequent inflammatory cow diseases like mastitis and common inflammations of the claws.

This paper discusses the main technical objective: the development of a precise temperature measurement for anatomic structures like the udder. The suggested method allows the quantification and compensation of disturbing influences of varying environmental conditions, drift and noise of the IRT sensor as well as inevitable movements of the animal. A novel combination of a referenced IRT with environmental conditions correction and an objective reproducible detection of anatomic structures by automatic image processing is suggested and investigated. This paper discusses the following aspects:

- modelling of the IR based measurement,

- uncertainty calculation after DIN 1319 [5] and optimisation for practical conditions

- model based correction of environmental influence factors and

- overview on image processing and feature extraction.

\section{Modelling}

A precise IR-measurement and practical working conditions necessitate the modelling and correction of disturbing influences. This model is the base for the evaluation of measurement uncertainty and gives potential for optimisation. DIN 1319 gives the following steps for the calculation of the measuring result: 
1. Describe the measurement as analytic model $f$, associate the input values $x$ with the measured value $y$ by an implicit or explicit function (1).

2. Prepare each input value $x_{i}$ by calculating the uncertainty $u\left(x_{i}\right)[5]$ or using a given value.

3. Calculate the complete measurement result composed of the measurement value $y$ and its uncertainty $u(y)$ solving the model function (1) and evaluate the combined uncertainty (2).

$$
\begin{aligned}
y & =f(\vec{x}) \\
u^{2}(y) & =\sum_{i=1}^{m} \sum_{k=1}^{m} \frac{\partial f}{\partial x_{i}} \frac{\partial j}{\partial x_{k}} u\left(x_{i}, x_{k}\right)
\end{aligned}
$$

\subsection{Measuring Task}

The generalized intention of the measurement is the quantification of the diagnostically relevant parameter $\Gamma^{1}$ by an IR based feature $M$. The measuring task can be divided into four models as shown in Fig. 1.

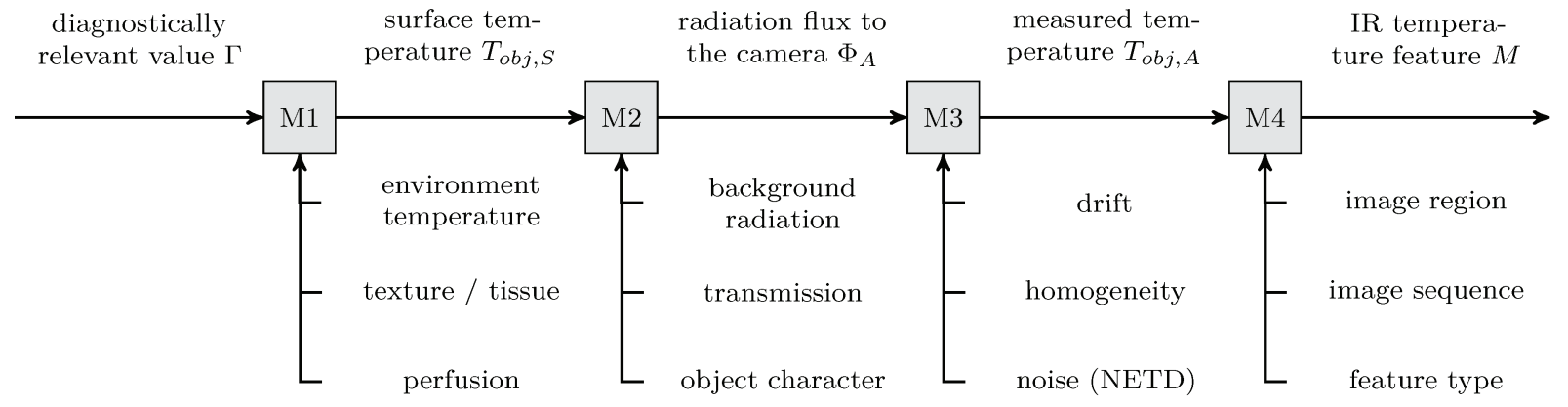

Fig. 1: Measuring chain and main influencing factors

The IRT only has the ability to measure surface temperatures, hence the first step M1 models the relation between the diagnostic relevant value $\Gamma$ and the surface temperature of the anatomic structure $T_{\text {obj,s. }}$. Due to the fact that $\Gamma$ is not directly observable a phenomenological model is discussed in section 2.4.

The next two steps describe the background radiation correction $\mathbf{M} 2$ and the properties of radiation measurement M3 of the IR camera, which are discussed in section 2.2.

In the last modelling step M4 the image points are related with the relevant anatomic structures (object detection) and advantageously merged into compact and robust (temperature) features $M$. This step is represented by the image processing and briefly discussed in section 2.3.

\subsection{Radiation Temperature Measurement}

The radiation temperature measurement (RTM) is divided into two parts represented by $\mathbf{M} 2$ and $\mathbf{M} 3$. The modelling step $\mathbf{M} 2$ describes the radiation flux effectively emitted by the object and the radiation detected by the camera. This encloses the emission characteristics of the object as well as interfering background radiation and the transmission characteristics of the atmosphere (here $\tau=1$ ). Based on the assumption of a homogeneous background at ambient temperature the detected radiation flux $\Phi_{o b j, A}$ can be modelled as linear combination of the black body radiation flux of the object $\Phi_{o b j, S}$ and the environment $\Phi_{a m b, S}$ [2]:

$$
\Phi_{o b j, A}=\varepsilon_{o b j} \tau \Phi_{o b j, S}+\left(1-\varepsilon_{o b j} \tau\right) \Phi_{a m b, S}
$$

The measured quantity $X$ of the IR camera is proportional to the radiation flux at the camera $\Phi$. The calibration links the quantity to the black body temperature by a look-up table. A parametric describing of $X$ could be fitted in step $\mathbf{M} 3$, estimating the parameters $K_{1} \ldots K_{4}[3]$ :

$$
\Phi \propto X(T)=f(T)=\frac{K_{1}}{\mathrm{e}^{\frac{K_{2}}{T}}-K_{3}}+K_{4}
$$

\footnotetext{
${ }^{1}$ The diagnostic relevant value appears as temperature change at the examined anatomic structure, which is in general not available for direct (conductive) measurement. Example: The temperature rise at an inflamed claw due to increased perfusion.
} 
For the modelling of the camera based uncertainty as drift and noise a linear model (5) consisting of an estimation of the true value $\hat{X}_{A}$ and three components of uncertainty is introduced:

- The time-depending ${ }^{2}$ uncertainty $X_{\Delta(t)}$ effects the image homogeneously. This stands approximately for the drift due to the self-heating of the sensor (Fig. 3).

- The time-invariant uncertainty $X_{\Delta(j)}$ for every pixel $j$ represents a fixed pattern image inhomogeneity (Fig. 2).

- The remaining time and pixel depending uncertainty $X_{\Delta(t, j)}$ includes the image noise and the model uncertainty.

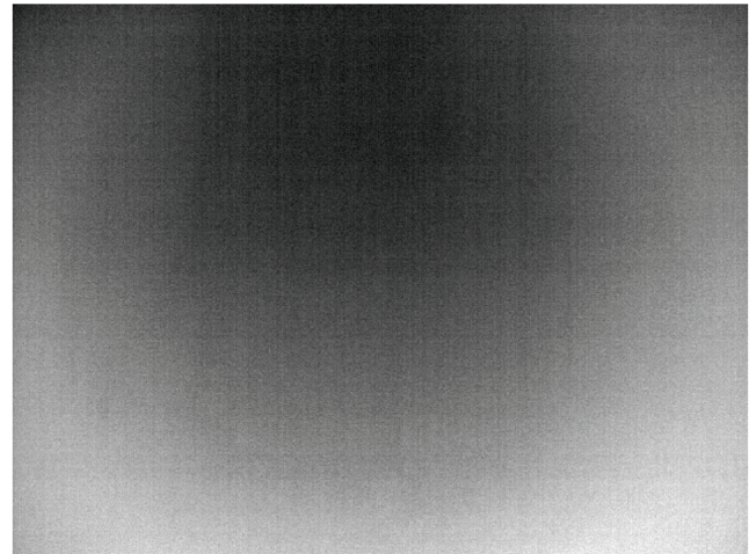

Fig. 2: Inhomogeneity of an IR camera

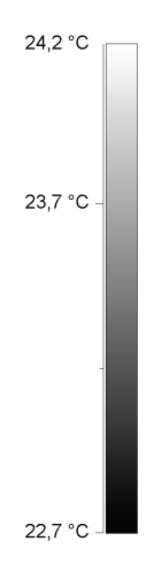

(1)



Fig. 3: Absolute value drift of a calibrated IR camera

This gives the actual combined measuring quantity $X_{A}$ :

$$
X_{\Lambda}=\hat{X}_{\Lambda}+X_{\Delta(t)}+X_{\Delta(j)}+X_{\Delta(t, j)}
$$

Summarizing the equations (3), (4) and (5) leads to a corrected image temperature $T_{o b j, S}$ (Fig. 4):

$$
T_{o b j, S}=f^{-1}\left(\frac{1}{\varepsilon_{o b j}} X_{o b j, A}+\left(1-\frac{1}{\varepsilon_{o b j}}\right) f\left(T_{a m b, M}\right)\right)
$$

For typical values of the measuring situation (see Table 1) and with equations (2) and (6) the resulting surface temperature uncertainty is $u\left(T_{o b j, S}\right)=2.1 \mathrm{~K}$. The main component of this uncertainty is $X_{\Delta(t)}$.

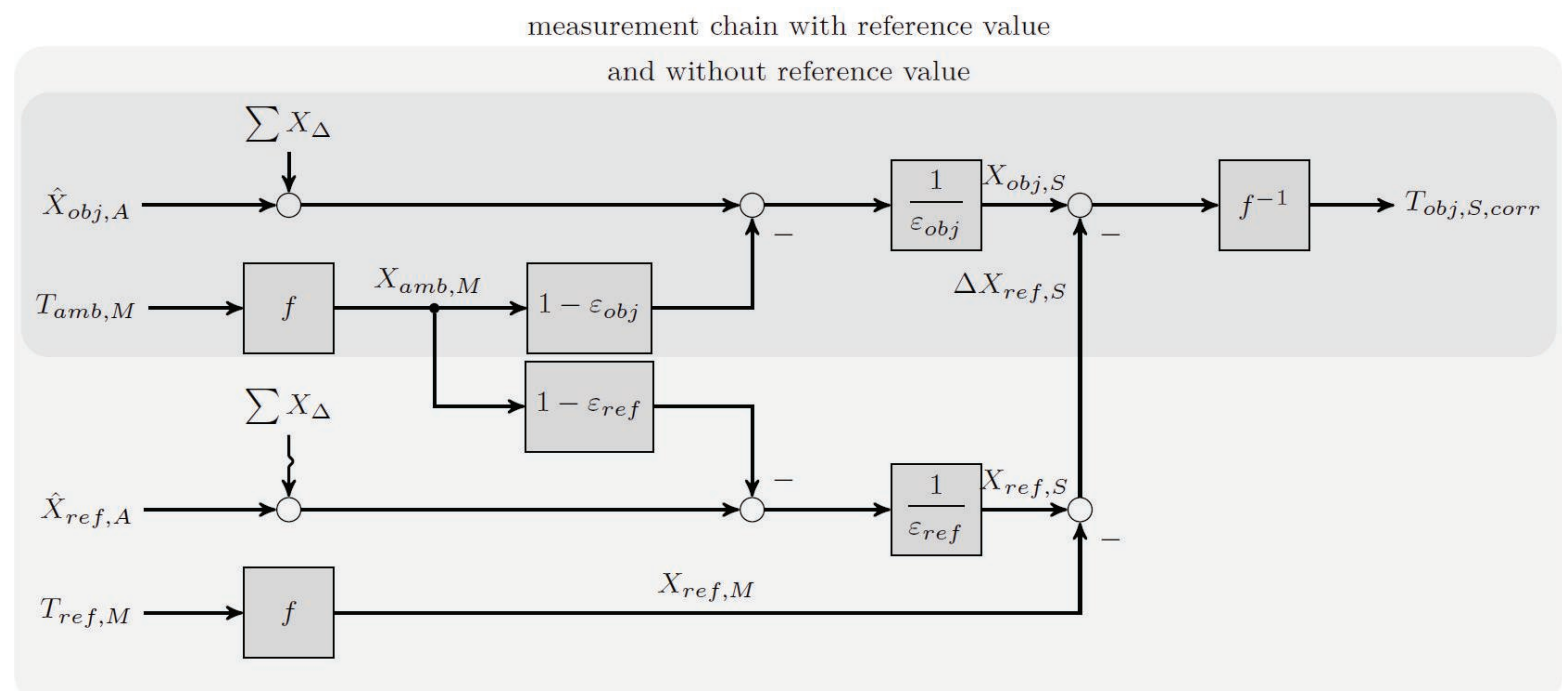

Fig. 4: Schema of the radiation temperature measurement with / without reference radiator

\footnotetext{
${ }^{2}$ The time dependence results from the recording of image series and effects e.g. the averaging or comparison of multiple images
} 
The resulting uncertainty is very high compared to the expected diagnostic temperature differences and the existing DIN-standard for IR clinical thermometers that claims an uncertainty $< \pm 0.3 \mathrm{~K}[6]$. The usage of a specialized reference radiator of known or measured temperature placed in the image area could reduce the uncertainty. The comparison of the IR temperature with the reference temperature allows the correction of the IR image (Fig. 4):

$$
T_{o b j, S, c o r r}=f^{-1}\left(\frac{1}{\varepsilon_{o b j}} X_{o b j, A}-\frac{1}{\varepsilon_{r e f}} X_{r e f, A}-\left(\frac{1}{\varepsilon_{o b j}}-\frac{1}{\varepsilon_{r e f}}\right) f\left(T_{a m b, M}\right)+f\left(T_{r e f, M}\right)\right)
$$

The equations (2) and (7) lead to a lower uncertainty of $u\left(T_{o b j, S, \text { corr }}\right)=0.35 \mathrm{~K}$ (Table 1), which is now dominated by the reference temperature uncertainty. That is $n$ not satisfying according to [6], but it allows the measurement of the ascertained diagnostic temperature differences of about $1 \mathrm{~K}$ [7].

Remark: The influence of the atmospheric transmission $(\tau=1)$ and the uncertainty of the characteristic parameters can be neglected.

Table 1: Input parameters and values for the calculation example

\begin{tabular}{ll}
\hline Parameter & Value and Uncertainty \\
\hline Object Temperature $^{3}$ & $\widehat{X}_{\text {obj, }}=\mathrm{f}\left(30{ }^{\circ} \mathrm{C}\right)$ \\
& $X_{\Delta(t)}=\mathrm{f}\left((0 \pm 2)^{\circ} \mathrm{C}\right)$ \\
& $X_{\Delta(j)}=\mathrm{f}\left((0 \pm 0.1){ }^{\circ} \mathrm{C}\right)$ \\
& $X_{\Delta(t, j)}=\mathrm{f}\left((0 \pm 0.08){ }^{\circ} \mathrm{C}\right)$ \\
Object Emissivity & $\varepsilon_{\text {obj }}=(0.965 \pm 0.0087)$ \\
Ambient Temperature & $\mathrm{T}_{\mathrm{amb}, \mathrm{M}}=(15 \pm 2)^{\circ} \mathrm{C}$ \\
\hline
\end{tabular}

\begin{tabular}{ll}
\hline Parameter & Value and Uncertainty \\
\hline IR Temperature of the & $\hat{X}_{\text {ref,A }}=\mathrm{f}\left(17^{\circ} \mathrm{C}\right)$ \\
Reference Radiator & $X_{\Delta(t)}=\mathrm{f}\left((0 \pm 2)^{\circ} \mathrm{C}\right)$ \\
& $X_{\Delta(j)}=\mathrm{f}\left((0 \pm 0.1)^{\circ} \mathrm{C}\right)$ \\
& $X_{\Delta(t, j)}=\mathrm{f}\left((0 \pm 0.08)^{\circ} \mathrm{C}\right)$ \\
Reference Emissivity & $\varepsilon_{\text {ref }}=(0.98 \pm 0.0029)$ \\
Reference Temperature & $\mathrm{T}_{\text {ref,M }}=(16 \pm 0.3)^{\circ} \mathrm{C}$ \\
\hline
\end{tabular}

The reference radiator yields two adjustable parameters:

- its temperature and

- its emissivity (material).

Both parameters affect the resulting uncertainty and should be selected properly. Analyses show that it is advantageous to choose the emissivity of the reference radiator equal to that of the object. The reference temperature shows the trend, that higher temperatures lead to higher uncertainty $\left(\mathrm{e} . \mathrm{g} . \mathrm{T}_{\text {ref }}=50^{\circ} \mathrm{C}\right.$ leads to $\left.u\left(T_{o b j, S, c o r r}\right)=0.46 \mathrm{~K}\right)$. With these assumptions and the practical boundary conditions an adapted reference radiator was developed and tested.

The model allows the examination of the sensitivity of RTM uncertainty for the relevant variability in environmental conditions. Fig. 5 shows the distribution of uncertainty over the ambient temperature for object temperatures $T_{o b j, S}$ of $(15 \ldots 40)^{\circ} \mathrm{C}$. For the relevant temperature span a RTM uncertainty lower than $0.47 \mathrm{~K}$ can be ensured.

Fig. 5: Resulting radiation temperature uncertainty for the typical input parameter range

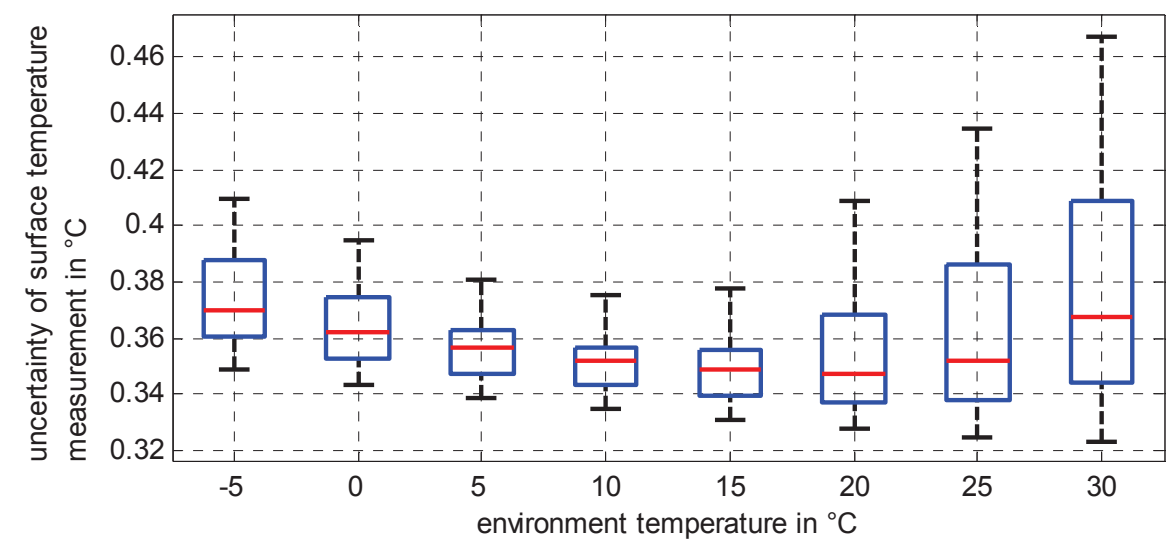

\footnotetext{
${ }^{3}$ Values are based on experimental results with the used camera and the specification of the manufacturer.
} 


\subsection{Image Processing}

In veterinary IR applications manual analysis is state of the art, an automatic solution is not known yet. Manual analyses are time consuming, affected by the know-how of the expert and not economic for large live stocks. An automatic IR image analysis therefore is an essential part of a health monitoring system. In the following a short overview is given of the developed image processing techniques (see also [13]) representing the modelling step M4.

In addition to several fixed model matching algorithms the variable shape approach Active-Shape-Model (ASM) was evaluated. After promising first results a modified ASM was developed with respect to the special characteristics of IR images and tested in several measuring campaigns. The final segmentation is based on the fitted model as shown in Fig. 6 . In this regions IR features are calculated. Features known from literature are the mean and maximal temperature. Within a systematic feature creation more than 35 values are derived and evaluated from each region.
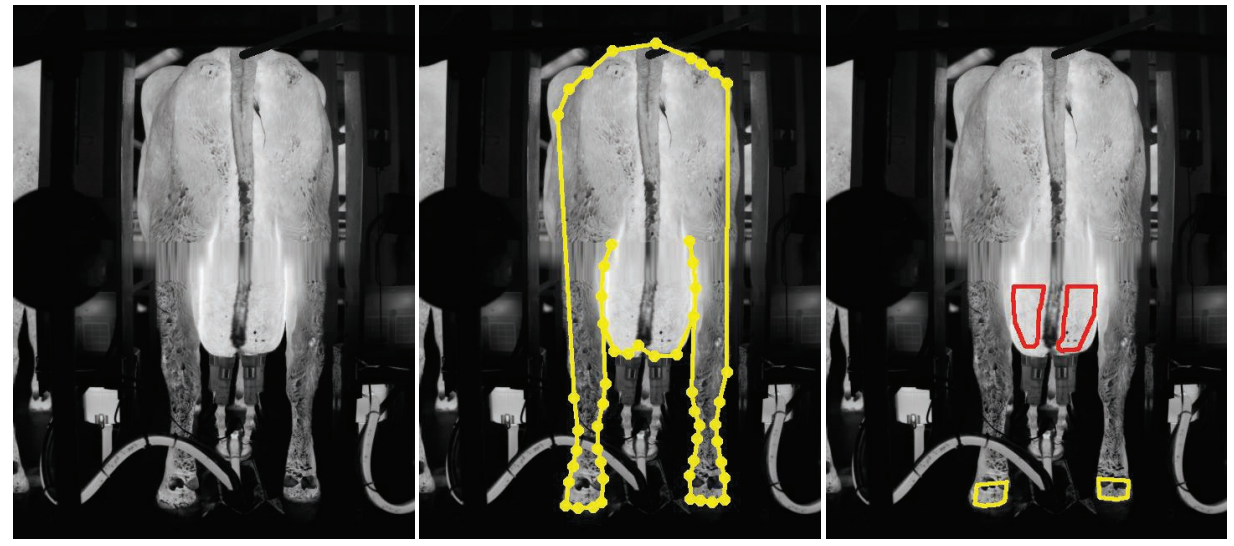

Fig. 6: Automatic image segmentation using the modified Acitve-Shape-Model approach (left: original image; middle: automatically fitted shape model; right: segmentation of claws and udder generated from shape)

\subsection{Diagnostic Temperature and Surface Temperature}

As mentioned earlier the diagnostic value $\Gamma$ is not directly measurable; for that reason no reference values are available and a phenomenological approach is used in modelling step M1. Based on the calculated IR temperature features possible influences as

1. environmental conditions (air temperature, humidity, air flow, irradiation),

2. physiological effects (oestrus, lactation, etc.),

3. individual different tissue and thermal coupling to surface temperature,

4. texture of the surface (esp. for coat) and

5. possible dirt and wetness

are modelled by their effect on the features. The environmental conditions (point 1) are logged separately by a climate measuring device providing the air temperature, humidity and pressure and an ultrasonic anemometer. Based on these data a regression model is estimated and corrected for each feature. The analyses show significant differences in robustness against the environmental conditions between the IRfeatures (table 2), witch allows a ranking of the features in combination with the diagnostic abilities.

Table 2: Correlation coefficients of the first $10 \mathrm{IR}$ features against selected environmental conditions

\begin{tabular}{llllllllllll} 
IR feature & 1 & 2 & 3 & 4 & 5 & 6 & 7 & 8 & 9 & 10 & $\ldots$ \\
\hline ambient temperature & 0.12 & -0.30 & 0.09 & 0.10 & 0.10 & 0.21 & 0.46 & 0.03 & 0.02 & 0.02 & \\
air pressure & -0.03 & 0.02 & -0.03 & -0.03 & -0.03 & -0.06 & -0.13 & 0.04 & 0.00 & -0.01 & \\
humidity & -0.04 & 0.10 & -0.03 & -0.04 & -0.04 & -0.10 & -0.21 & 0.04 & 0.00 & 0.00 & \\
\hline
\end{tabular}

Physiological effects and individual differences are transferred to the animal individual diagnostic step that is not discussed here. The last two points have to be handled by the image processing in a way that the calculated features are robust against these unavoidable influences or the disturbance is detected 
and the affected images are rejected for the analysis. The investigations on model adaptation and the integration in the uncertainty calculation are ongoing.

\section{Summary and Outlook}

Most veterinary IRT studies neither quantify the measurement uncertainty nor discuss it in relation to the determined diagnostically relevant temperature differences.

The analysis shows that the uncertainty of common IRT studies is greater than $2 \mathrm{~K}$, which is a lot compared to the relevant differences. Using a state of the art IR camera and an optimized reference radiator the uncertainty could be reduced to below $0.47 \mathrm{~K}$. The developed reference radiator satisfies the practical requirements of veterinary applications proven in large field studies. This clearly increases the comparability and repeatability.

The introduced model of the radiation temperature measurement can be used for the evaluation of veterinary IR investigations under practical conditions. By comparing the calculated uncertainty and the diagnostic temperature difference the applicability can be determined. Furthermore, the uncertainty can be used as quality criterion for the diagnostic algorithms.

The investigations concerning the diagnostic abilities and the robustness of the IR features are not finished yet. The integration of uncertainties arising from the image processing into the described model is another goal.

With the precise IR measuring system and the automatic image processing two essential parts of an automatic veterinary health monitoring system were developed.The framework provides a rapid, automatic processing for a multitude of IR images, the online processing seems achievable as well as the transfer to medical applications.

\section{Acknowledgement}

This investigations are done within the scope of the VIONA project financed by the German Federal Ministry of Education and Research (BMBF, ID 03WKP04B). The authors are grateful to the industry partners of the project DIAS Infrared, Ralle Landmaschinen, and Yoo $\mathrm{GmbH}$ as well as the hosting farms Methauer Agro AG and Großdrebnitzer Agrar GmbH.

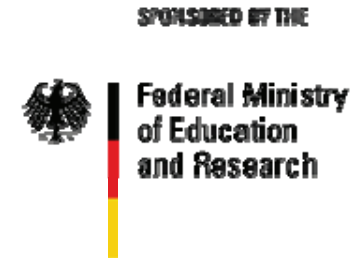

[1] Barth K.: Basic investigations to evaluate a highly sensitive infrared thermograph-technique to detect udder inflammation in cows, Milchwissenschaft 55 (2000)

[2] Bernhard, F. [Ed.]: Technische Temperaturmessung, Springer Verlag, 2004

[3] Budzier, H. and Gerlach, G.; Thermische Infrarotsensoren; WILEY-VCH; 2010

[4] Colak A., Polat B., Okumus Z. Kaya M., Yanmaz L. E., Hayirli A.: Short Communication: Early Detection of Mastitis Using Infrared Thermography in Dairy Cows, J. Dairy Sci. 91 (2008)

[5] DIN 1319-4, 1999. Fundamentals of metrology - part 4: Evaluation of measurements; uncertainty of measurement. - Berlin : Beuth Verlag

[6] DIN EN 12470-5, 2003. Clinical thermometers - part 5: Performance of infra-red ear thermometers. - Berlin : Beuth Verlag

[7] Franze, U.; Geidel, S.; Wirthgen, T.; Zipser, S.: Infrarot - Thermographie bei Milchkühen, 11. Jahrestagung der WGM (2010)

[8] Gschröderer, Ch.; Möglichkeiten der Integration der Thermographie in den Routineablauf einer Rinderbesamungsstation; masters thesis; University of Applied Sciences Weihenstephan; 2006

[9] Hilsberg, Sabine; Aspekte zur klinischen Anwendung der Infrarot-Thermographie in der Zoo- und Wildtiermedizin; dissertation; Universität Leipzig; 2000

[10] Hovinen M., Siivonen J., Taponen S., Hänninen L., Pastell M., Aisla A.-M., Pyörälä S. (2008): Detection of Clinical Mastitis with the Help of a Thermal Camera, J. Dairy Sci. 91 (2008)

[11] Knizkova, I.; Kunc, P. et al.: Application of infrared thermography in animal production, J. of Fac. of Agric., OMU, 2007,22(3)

[12] Lawson, R.N.: Implications of surface temperature in the diagnosis of breast cancer, Canad. med. Ass. J. 75, S. 309-318, 1956

[13] Wirthgen, T.; Zipser, St.; Geidel, St., Franze, U., Dietel, F. and Alary, Th.; Active Shape Segmentation- an Application for a Precise Body Temperature Measurement of Dairy Cows; SCIA 2011; (submitted paper) 